\title{
Infrared Inspection of Steel Structures Under Load
}

\author{
by Barbara Szymanik and Grzegorz Psuj
}

\author{
Department of Electrical and Computer Engineering, Faculty of Electrical Engineering, West Pomeranian University of \\ Technology, al. Piastów 17, 70-310 Szczecin, Poland, szymanik@zut.edu.pl
}

\begin{abstract}
This paper presents in detail the experimental methodologies and the results of the infrared investigation of the steel samples under load. Moreover the second, electromagnetic method (EM) for non destructing evaluation was used for monitoring the fatigue process. The IRT allows to observe the general global condition of the material while the EM to carry out local, detail examination of the fatigue stage in critical region. The presented inspection results are compared and discussed.
\end{abstract}

\section{Introduction}

The dynamic development of many modern industries is associated with the production of the steel structures with a high level of complexity. It is expected that these structures meet the highest standards of safety. However, maintaining relatively low manufacturing costs are also crucial. The success of many companies and corporation is mainly based on the possibility of the long term and low cost product usage. Therefore the continuous and systematic monitoring of the changes in the material properties of the steel structures is very important. The crucial feature of such a monitoring is the necessity of observation the structure under load.

The estimation of fatigue failure risk requires to carry out the analysis and prediction algorithms taking into consideration initial strength capacity, production technology and types of possible flaws introduced during production or operation period [1]. The process requires also determination of local condition of the material, which involves evaluation of its microstructure, applied and residual stress values in accordance to strain state as well as crack grow area within the examined region. Therefore, the need for application of nondestructive testing and evaluation methods contributing the process of determining the state of stress and damage in the material emerges [1, 2].

There are many methods, that can be useful in steel structures monitoring (electromagnetic, magnetic, radiographic and ultrasound). In the recent times the increased importance of thermographic method may be also observed [3]. Active infrared thermography is one of the methods that are widely used in the non-destructive testing of materials. Internal structure of the tested material, in particular defects (such as cracks or weakening of the structure caused by the mechanical stress) affect the free flow of heat. Sensitive infrared camera is used to observe the temperature distribution on the sample's surface. The high temperature resolution allows to observe even small internal inhomogeneities in the form of the overheated or colder places, showing the location, size and type of defects.

The purpose of this paper is to present and compare the results of fatigue monitoring carried out by electromagnetic and infrared thermography method [4].

\section{Experimental methods and systems}

In the process of assessing the degradation state of the material several factors are considered crucial. It is important to analyze what type of defects may occur in examined construction. Furthermore, the possibility of carrying out a rapid assessment of the overall condition of the structure as well as obtaining detail information for the selected critical areas has to be also considered. Therefore in this paper two nondestructive testing methods are used to monitor the progress of fatigue process in steel samples. Electromagnetic method for the local assessment and infrared thermography for global investigation.

In the global assessment the most important is that the infrared camera with a high resolution allows the simultaneous observation of relatively large areas. The observation allows the initial location of the areas where the material is subjected to higher loads. Another advantage of this method is the ability to quickly inspect the materials due to the fact that the ferromagnetic materials have generally a high thermal conductivity coefficient. After preliminary location of the areas susceptible to damage, the thermal camera with the macro lens can be used for the final, local assessment of the steel structure.

The temperature changes of the examined sample were observed by the infrared camera FLIR A325 (4). The camera has the temperature resolution up to $0.05{ }^{\circ} \mathrm{C}$ and is able to record 60 thermograms per second.

Due to the electromagnetic properties of steels utilized for the constructions, the use of electromagnetic testing methods becomes a natural solution for evaluation of theirs damage stage. Material properties are influenced by the stress and fatigue loading conditions. It is known that microstructure features affect the magnetic one [2]. Therefore, by monitoring changes of magnetic properties, the damage state of the material can be evaluated. Various magnetic 
properties can be considered in the process of damage state evaluation [2, 5]. Hysteresis loop parameters such as remanence or coercivity as well as Barkhausen noise (BN) characteristic values such as signal's energy shows good correlation with the damage state of the material.

In order to monitor changes of electromagnetic properties a transducer (2) allowing BN observation was used. The transducer (Fig. 2) consists of excitation coil and two pick-up coils. The excitation coil is wound on the C-shaped ferrite core. The details of the EM subsystem and transducer can be found in [6]

Measurements were carried out using a computerized universal NDT system (Fig.1.). The system contains of two main subsystems: a fatigue stress unit (FSM) and a nondestructive inspection one (IRT + EM). In order to proceed the cyclic loading tests, a hydraulic type machine (1) was used. In order to synchronize operation, both subsystems were managed and controlled by a PC class computer (5) equipped with data acquisition boards NI 6221 and NI 6251 using a dedicated software implemented in LabVIEW environment. All experiments were carried out for two planar specimens made of low carbon construction steel St3s (PN-88 H-84020). The shape and dimensions as well as photo of the samples are presented in Fig. 3. During the experiments the samples were loaded in a longitudinal direction using a cyclic loading machine. The maximum loading strength was set to $380 \mathrm{MPa}$. In the initial phase of loading process the samples were subjected to preliminary tension using the constant stress value of $220 \mathrm{MPa}$. In the next stage the alternating cycling loading of frequency equal to $4 \mathrm{~Hz}$ was added to the offset value. In order to initiate the fatigue crack grow in the selected part of the sample, a notch of $1 \mathrm{~mm}$ length was made on one side of the sample in the central part of region of interest (ROI). The data acquisition was carried out in periods. Every 240 fatigue cycles both BN signals and IRT camera images were collected and stored. The total number of fatigue cycles obtained during experiments were close to $2 \cdot 10^{5}$ in case of sample $s 1$ and $1 \cdot 10^{4}$ in case of sample s2.
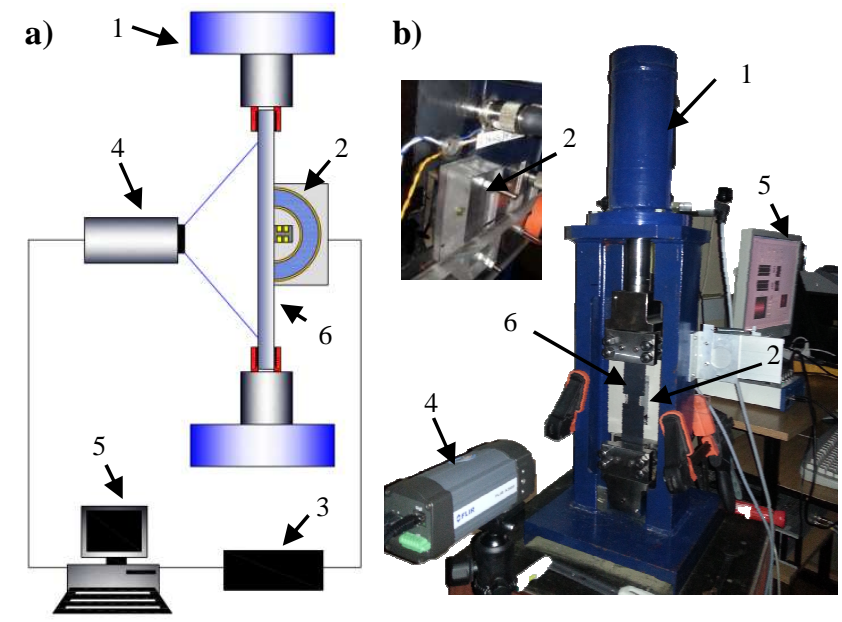

Fig. 1. Measuring system: a) schematic diagram, b) photo; 1 - fatigue stress machine, 2 - electromagnetic transducer, $3-$ electromagnetic excitation and pickup subsystem, 4 - Infrared camera, 5 - PC controlling and acquisitioning computer, 6 - steel sample.

a)

b)
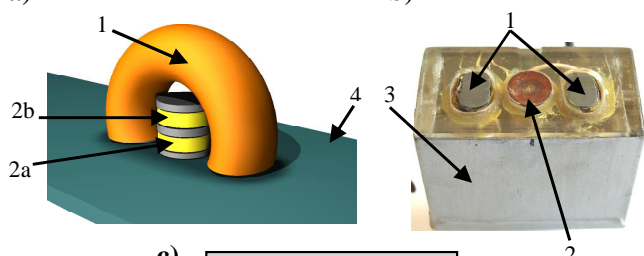

c)

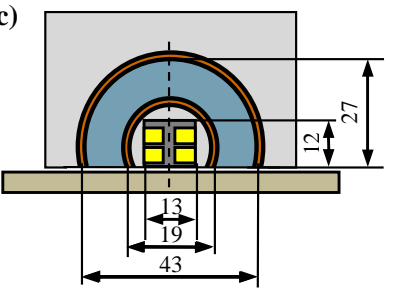

Fig. 2. Barkhausen noise transducer: three-dimensional view (a), photo (b) and cross-section view with dimensions (c) of the transducer; 1 - ferrite core, 2a- main pick-up coil, $2 \mathrm{~b}$ - reference pick-up coil, 3 - shielding, 4 - examined sample 
a)

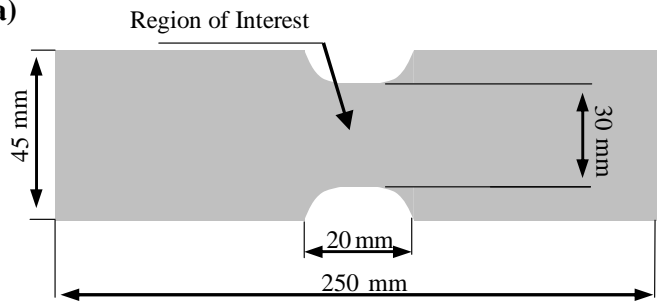

b)

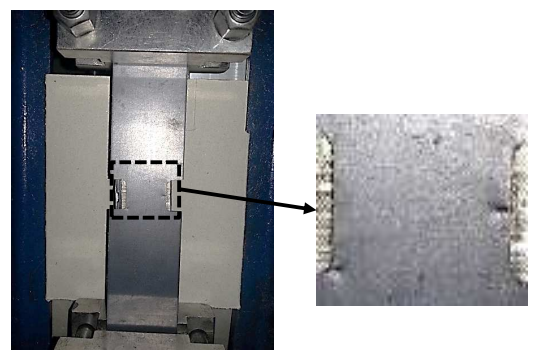

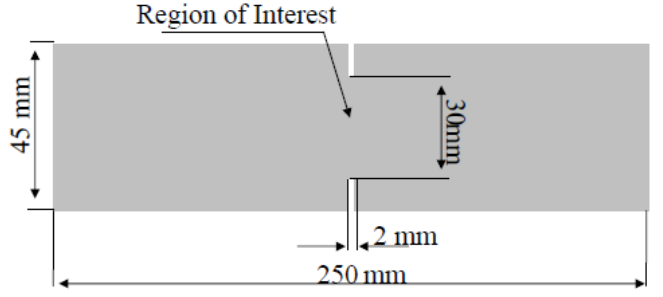

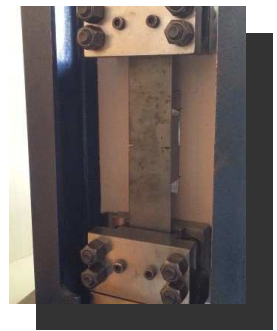

Fig. 3. View of fatigue loaded steel samples: a) draw with the shape and size of the samples (left s1 and right s2), b) photoes of the samples under fatigue test with enlargement of depicted (by black rectangle) region of interest

\section{Signal processing methods and results}

In case of IRT, the obtained thermograms sequence was analysed by comparing the temperature values from two chosen regions: sound region and region of interest (shown in Fig. 4). First all the thermograms in the sequence were normalised by subtracting the computed mean value thermogram. This procedure allows to remove the temperature background. The maximum and standard deviation values were compared for both regions. Moreover the IR thermography gives also the possibility to visualize the temperature distribution directly at the sample's surface. The sequence of thermograms was processed using Discreet Fourier Transform (DFT). As a result the sequences of phaseograms and amplitude images were then obtained.

In case of BN, first several features were extracted directly from BN signal. The analysis of characteristic values as well as statistical ones were proceeded. As a result, parameters such as standard deviation, variance or energy were obtained. Next, the envelope of BN signals were computed. The moving window median filtration was applied in order to get the BN signal profile. Then the 5th order polynomial was fitted to achieve the BN envelope. Finally, the features such as envelope's peaks value, position or width were calculated.

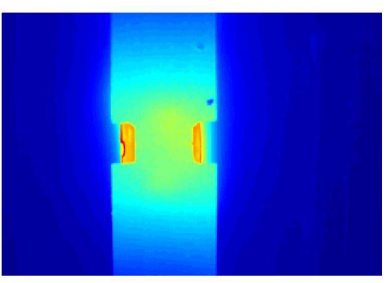

a)

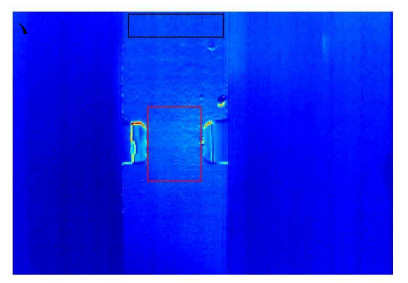

b)

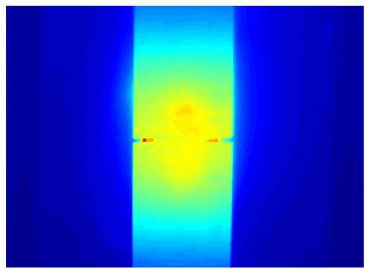

c)

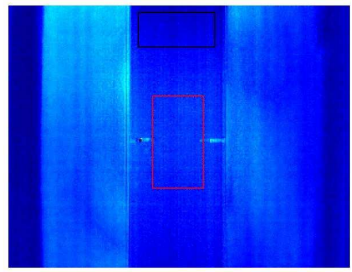

d)

Fig. 4. Exemplary thermograms of both samples (s1 and s2) a), c) and thermogram after normalization with marked sound region (black rectangle) and area of interest (red rectangle) b) and d).

Generally the fatigue process can be divided into three stages: microstructural change ending with a fatigue breakthrough stage, development of crack growth area in the material, and finally destruction of the material [2].

First, the sample $\mathrm{s} 1$ was analyzed. In case of infrared thermography the comparison between the maximum and standard deviation regions from selected regions are shown in Fig. 7 a) and b). The red lines show the parameters' values in the region of interest. Both parameters are significantly different from the sound area (shown as the blue lines in both cases). One can easily notice, that in case of IRT it is possible to distinguish mentioned three characteristic stages of fatigue process. Especially in case of standard deviation the initial grow of parameter is visible during the stage of microstructual change. This stage of rapid change is short (lasts till around $10^{3}$ loading cycles) and then the signal stabilize, which is the indication of micro crack growth stage. The final stage, when the rapid crack growth is observed is 
indicated by the large change of signal (starts around $2 * 10^{5}$ loading cycles), marked with green rectangle in Fig. 7 and Fig. 8.

a)

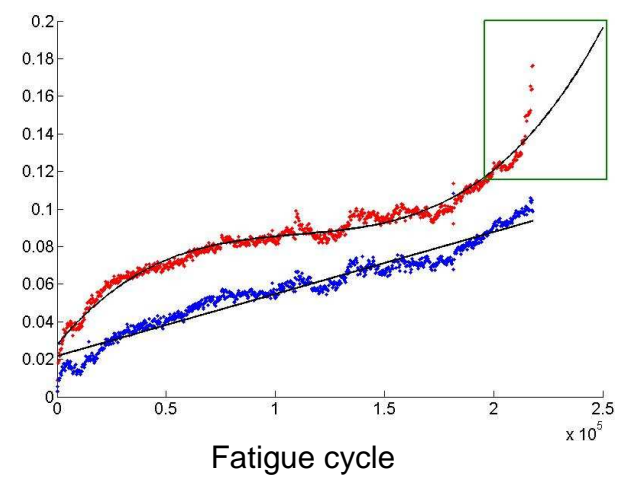

b)

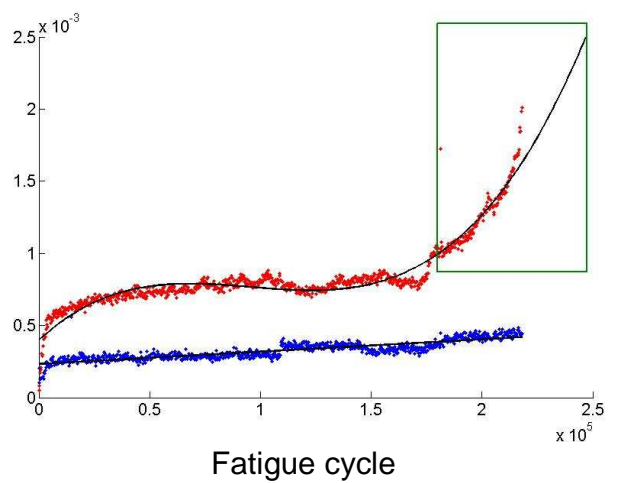

Fig. 7. The comparison of the maximum a) and standard deviation b) values from the sound region (blue line) and region of interest (red line). The rectangle marks the crack generation indication

The crack visualization using IR was also possible. In case of thermography, the time sequence of thermograms was recorded during the fatigue process. It is possible to use DFT (discreet Fourier transform, the method diagram is shown in Fig. 8) to obtain the sequences of phase and amplitude images from the time sequence. This procedure is useful in case of observation the dynamic changes in the image - like rapid growth of the crack in this case. The Fig. 9 show the amplitude and phase images chosen from the thermograms' sequence processed with DFT. The heat transfer within the defected area is significantly disturbed, thus the crack is visible as the border between heated and unheated area. The hot spot visible in both images is produced partially by BN transducer.

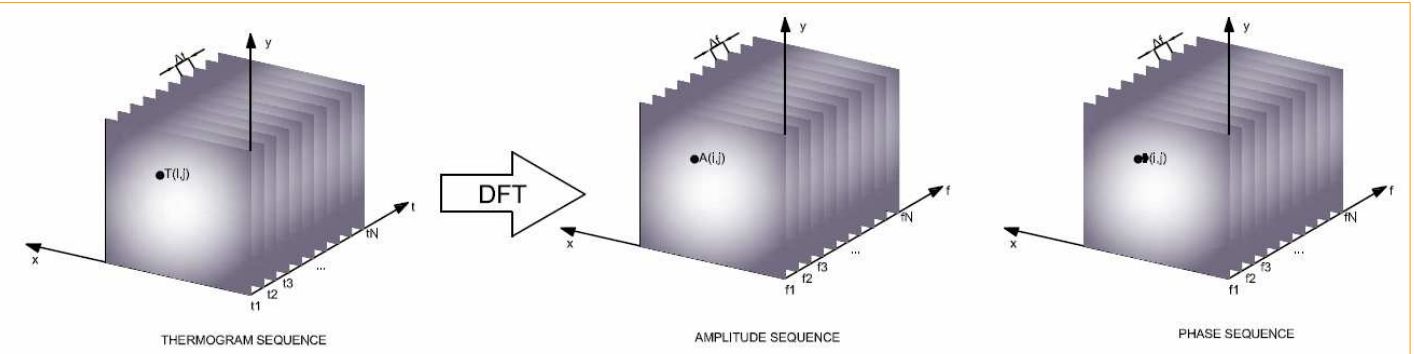

Fig. 8 Discreet Fourier transform diagram

a)

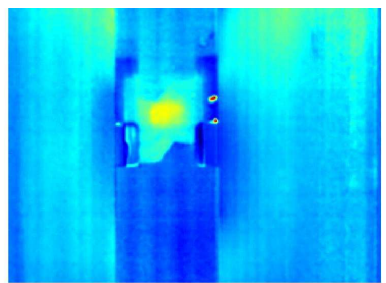

b)

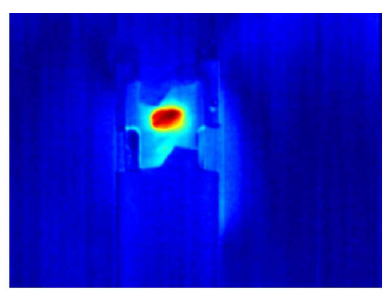

Fig. 9. The phase a) and amplitude b) images of the sample with crack generated in the load process

In case of BN, the distributions of signals peak position and median value of BN burst envelope are shown (Fig. 10). Similar to pervious observation using IRT, at the beginning of the fatigue process (until $2.5 \cdot 10^{3}$ of fatigue cycles) one can notice significant change of parameters value with cycles which is especially visible on plots of peak position and median value. The detail views of this part are presented in Fig. 6. The observed behaviour can be explained by the process of microstructural changes resulting in material softening [7]. This stage is relatively short and material properties stabilize after $10^{3}$ of loading cycles. The second stage, in which the micro-cracks growth occurs, lasts most of the time of whole fatigue process. During this part Barkhausen noise parameters do not change significantly, however there can be seen week trends in achieved distributions. Final stage (begins around to $2 \cdot 10^{5}$ of fatigue cycles) corresponds to rapid growth of the crack area and final fracture of the material. This can be also noticed by significant change of trends on the obtained parameters distributions. 
a)

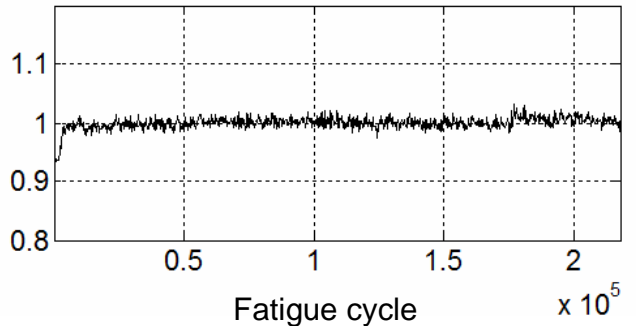

c)

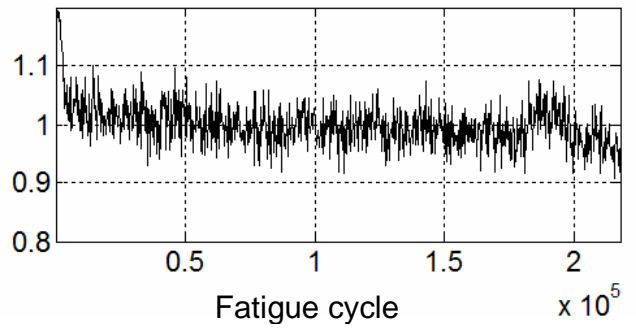

b)

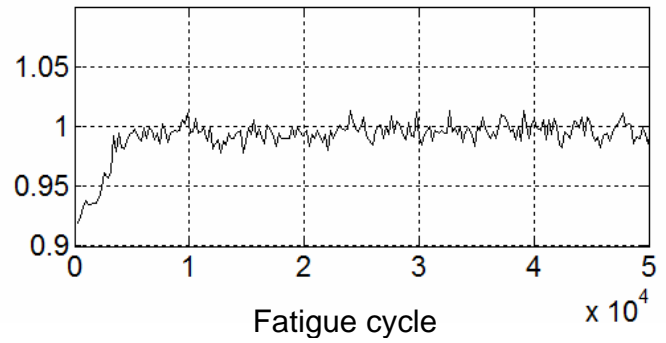

d)

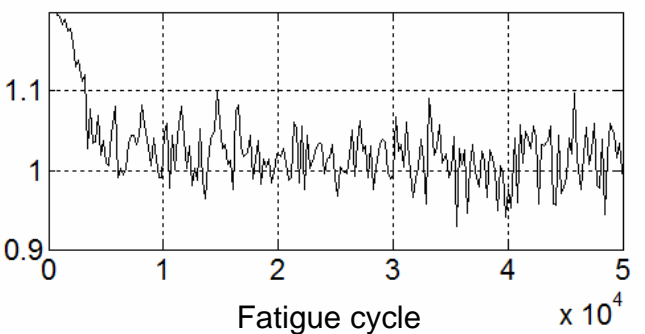

Fig. 10. Selected results obtained using Barkhausen noise method: a) envelope peak position, b) envelope peak position achieved at the beginning of the fatigue process c) envelope's median value, d) envelope's median value achieved at the beginning of the fatigue process; all distributions were normalized by the mean value of the parameter

Similar tests were conducted using sample s2. The region of interest in this sample is much smaller in comparison with sample s1 (only $2 \mathrm{~mm}$ of width, as indicated in Fig. 3). It was interesting, to find out if also in this case the observations' results will be similar, and recognition of all three stages of fatigue process will be possible using both NDT methods.

a)

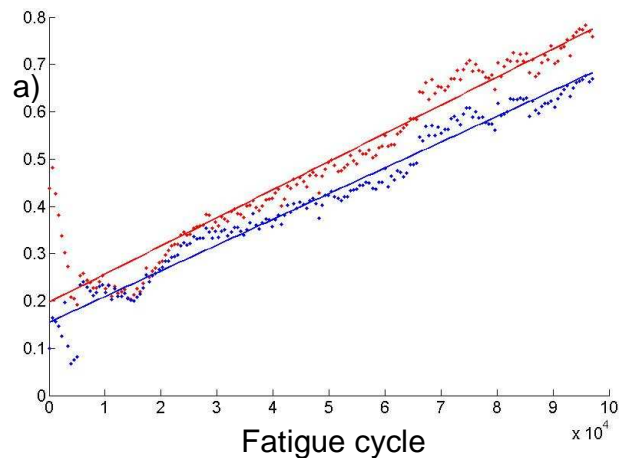

b)

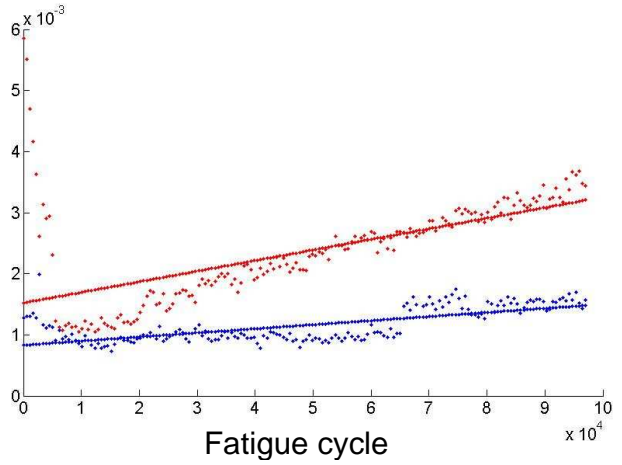

c)

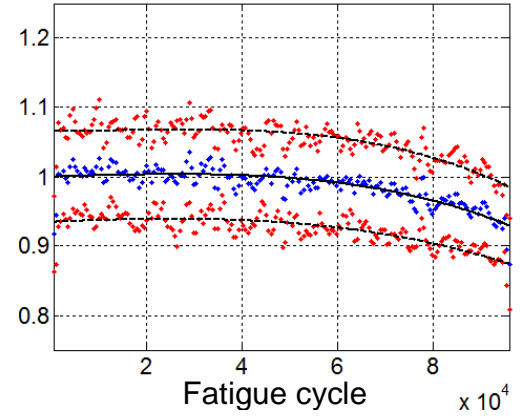

Fig. 11 The maximum a) and standard deviation b) values obtained for using IRT in sample s2 (red line: region of interest, blue line: sound area), c) the envelope's median value for BN signal (blue line - the envelope's median value, red lines - \pm 1 SD)

The results of IRT observations are shown in Fig. 11. Similar to the previous sample, here also the comparison between maximum and standard deviation values between the region of interest and sound area are shown. However in this case in the region of interest the significant drop of the both signals' values in the first stage of fatigue process is observed. Then the signals stabilise (around $1 \cdot 10^{4}$ fatigue cycle). There is no significant difference between the sound 
area and region of interest signals up to around $3 \cdot 10^{4}$ fatigue cycle. Then the difference arises which may indicate the beginning of the last stage of fatigue process leading to sample destruction.

This visible different behaviour (in comparison with s1) is also visible in BN signal. Here the envelope's median value is presented. The significant drop of the signal trend is visible, especially in the last stage of fatigue process.

It is quite difficult to interpret the results at this stage. The IRT signals' growth at the last stage of fatigue process correlate with our expectations. However the signal value drop in case of BN shows the significant difference of the both samples behaviour. In further research we will focus of the more local examination of the sample, using macro focus in IRT and smaller transducers in EM method.

\section{Conclusions}

In this paper we presented the experimental methodology and results of inspection of steel samples under load. The main inspection method was infrared thermography with the support of Barkhausen noise analysis technique. The selected methods allows to observe the fatigue process by means of different physical aspects (e.g. changes of magnetic and thermal properties during fatigue). Thereby making it possible to obtain a wider range of information about the state of the structure. As it was mentioned the interpretation of the results is complicated. The further work will be focused on providing the methods of local assessment of the sample, and integration of data gathered.

\section{REFERENCES}

[1] Dobmann G., Ciclov D. C., Kurz J. H., NDT and fracture mechanics. How can we improve failure assessment by NDT? Where we are - where we go?, Insight, Vol. 53, No. 12, 2011

[2] Palit Sagar S., et al., "Magnetic Barkhausen emission to evaluate fatigue damage in low carbon structural steel", International Journal of Fatigue, vol. 27, 2005, pp. 317-322

[3] Maladegue X., Theory and practice of infrared technology for nondestructive testing. JohnWiley and Sons, 2001.

[4] G. Psuj, B. Szymanik: Fatigue Monitoring of Steel Structures using Electromagnetic and Infrared Thermography Inspection Methods, Proceedings of ISTET 2015, Kołobrzeg, Poland

[5] Psuj G., Chady T., Enokizono M., Observation of material degradation under fatigue and static loading condition using selected electromagnetic NDT methods, Material Science Forum, 721, 120, 2012, 120-126

[6] Chady T., Psuj G., Fuzja elektromagnetycznych metod testowania w zastosowaniu do wizualizacji zmian materiałowych w stalowych elementach poddawanych naprężeniom, Przegląd Elektrotechniczny (Electrical Rev.) R.82, Nr 1/2006, pp.1-7

[7] Lindgren, Lepisto T., "Effect of cyclic deformation on Barkhausne noise in mild steel", NDT and E International, 36 (2003), pp. 401-409. 